\title{
Scrambler (Calmare) Therapy for Intractable Chronic Pain
}

\section{Soo A Kim}

Department of Physical Medicine and Rehabilitation, Soonchunhyang University Cheonan Hospital, Soonchunhyang University College of Medicine, Cheonan, Korea

Intractable chronic pain is a debilitative pain conditions for most patients. Unfortunately, the pain control managements currently have shown that it is not appropriate to reduce pain and limited efficacy and sometimes unfavorable in some patients. Scrambler (Calmare) therapy, used as an alternative treatment for chronic neuropathic pain recently, is a noninvasive approach to relieve pain by changing pain perception. The author reports the clinical experiences on effectiveness of Scrambler therapy for three patients with intractable pain refractory to conventional treatment.

Keywords: Scrambler therapy; Intractable pain; Conventional treatment

\section{INTRODUCTION}

Chronic neuropathic pain is often difficult to treat effectively. Although conventional treatments including medication such as opioids and antipsychotic drugs, physical therapies and other managements can help, but some has side effects and limited effectiveness. Scrambler (Calmare) therapy is a noninvasive approach to pain control that attempts to relieve pain by providing 'non pain' information via cutaneous nerves to block the effect of pain information $[1,2]$. There are some research showed that Scrambler therapy has relieved refractory chronic pain in several uncontrolled clinical conditions; cancer pain with abdominal pain [1], neuropathic pain including failed back surgery, brachial plexus neuropathy and others [3], post herpetic pain [4,5], chemotherapy-induced neuropathic pain [6-8], cancer-related pain $[8,9]$, chronic neuropathic pain including postsurgical pain and spinal canal stenosis [2], and low back pain [10]. In this case reports, scrambler therapy was applied to patients who had difficulty controlling pain with other conventional treatment methods.

\section{CASE REPORTS}

\section{Case 1}

A 54-year-old female patient visited outpatient clinic with pain in right hip area, which starts 84 months ago. She suffered from constant throbbing pain with intermittent burning and electricshock like pain. Several years ago, she complained for upper and lower extremities' weakness and numbness and was diagnosed chronic inflammatory demyelinating polyneuropathy. She had taken several oral medications including pregabalin $600 \mathrm{mg}$ and prednisolone $20 \mathrm{mg}$ per day for several years. Seven years ago, she felt right hip pain and radiologic finding showed the suspicious of benign chondroma in right femoral intertrochanteric area. She had been treated with local intralesional lidocaine injection (sometimes steroid injection), epidural block, transcutaneous electrical nerve stimulation, extracorporeal shockwave therapy (ESWT), and opioid medications. However, her symptoms were gradually aggravated in spite of all treatment and the pain score on the visual analog scale (VAS) was 9/10. So we rechecked the radiologic finding of pelvic area and hip, the magnetic resonance imaging (MRI) findings showed mild subtrochanteric bursitis on both hips (Fig. 1). 

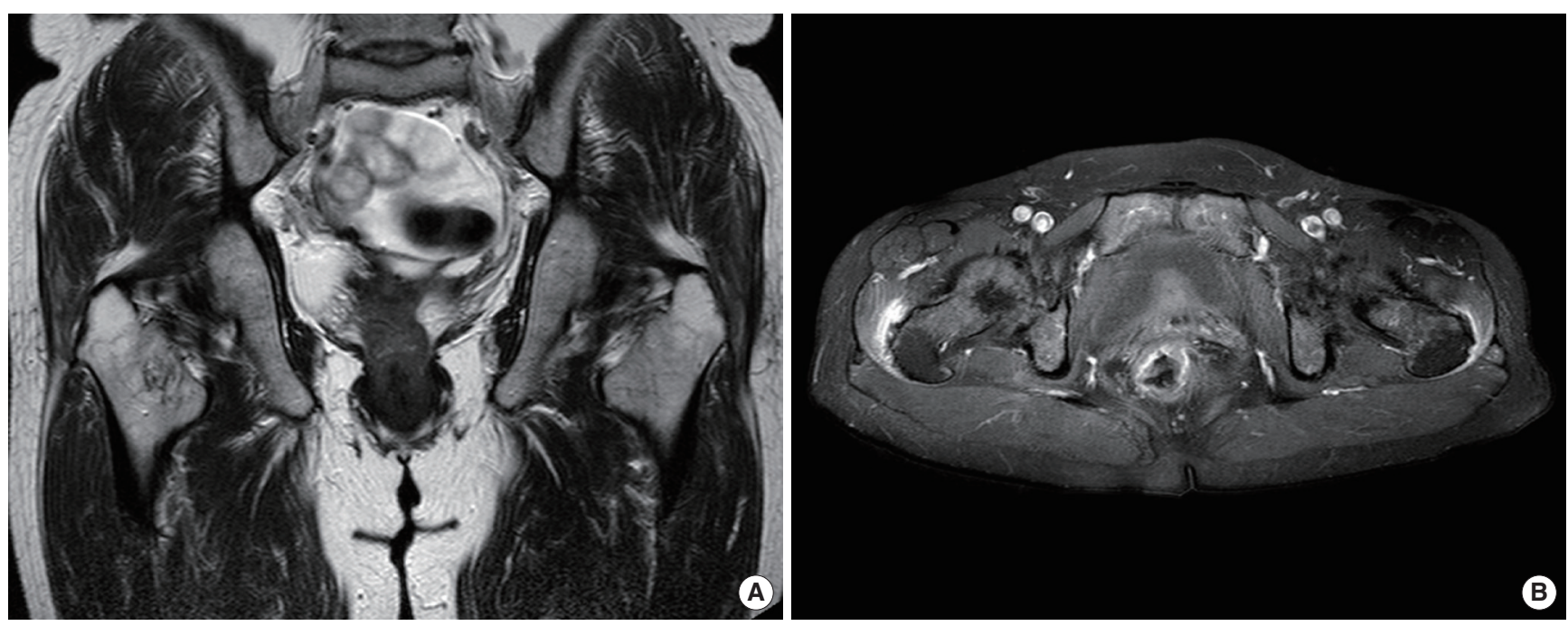

Fig. 1. (A) Coronal T1 weighted and (B) sagittal T1 weighted magnetic resonance imaging image showed mild subtrochanteric bursitis on both hips.

Thus, we considered the use of Scrambler therapy for pain relief. Under informed consent, the patient was treated for 50 minutes daily for 10 consecutive days. The electrode patches attached the symptomatic area including right thigh and buttock and electrode intensity was increased to $5.5 \mathrm{~mA}$ until she endured. The impulses are transmitted by surface electrodes placed on the skin and the electrical charge used is low and even though the highest setting, the maximal intensity is $5.5 \mathrm{~mA}$. A scrambler therapy was initiated and the VAS score was 9/10. After second session, the VAS score was $7 / 10$ and she reported that the throbbing and tingling sensation decreased to a tolerable range. After fourth session, her pain score was 3/10 and the last treatment showed VAS score to 2/10. Six month later, she visited outpatient clinic and her VAS score was $3 / 10$ and satisfied with her conditions.

\section{Case 2}

A 56-year-old female patient visited outpatient clinic with pain around both ankle and foot, which started several years ago. Ten years ago, she was diagnosed Guillain-barre syndrome and transverse myelitis on thoracic level after lower extremity weakness and numbness. She suffered from intractable pain on dorsal foot area and took pain control medications including neurontin $1,800 \mathrm{mg}$, alprazolam $1 \mathrm{mg}$, oxycodone $40 \mathrm{mg}$, and zolpidem $6.25 \mathrm{mg}$ per day. And whenever she felt abrupt pain attack intermittently, she took intramuscular diclofenac injection and intravenous tramadol infusion, sometimes fentanyl patch used but not appropriately reduced pain. She did not want further medication and invasive procedures. Thus we treated her with Scrambler therapy. The 10 electrode patches was attached both her foot dorsum and ankle area following L5 and S1 dermatome distribution and electrode intensity was increased until she endured. With the patient's informed consent, we started the Scrambler therapy with a 50-minute daily treatment for 10 days. At the beginning, the VAS score was $7 / 10$. After seventh session, the VAS score was $4 / 10$. On the last day, the VAS score was 3/10 and the abrupt pain attack was reduced to a tolerable range. Three months later, at the patient's follow-up visit, the VAS score was 4/10 and the frequency and intensity of abrupt pain attack was decreased to $50 \%$. She satisfied her condition these days.

\section{Case 3}

A 76-year-old female patient complained her mid thoracic area pain for three years ago. The pain was characterized as a splitting pain around the mid thoracic area. She had a lumbar discectomy operation 15 years ago in her past history. Radiologic finding showed multiple old compression fracture due to senile osteoporosis (Fig. 2). She had a several management for pain control including trigger point injection, epidural block, physical therapies, and ESWT. She still complained her pain even though various management. Thus we treated her with Scrambler therapy. Under the patient's informed consent, we treated with the Scrambler therapy with a 50 -minute daily treatment. The 10 electrode patches were attached vertical placement following the paraspinalis muscle. The electrode intensity was increased until she endured. At the beginning, the VAS score was 8/10. After third session, the VAS score was $5 / 10$. She finished her treatment after five treatments. Four weeks 


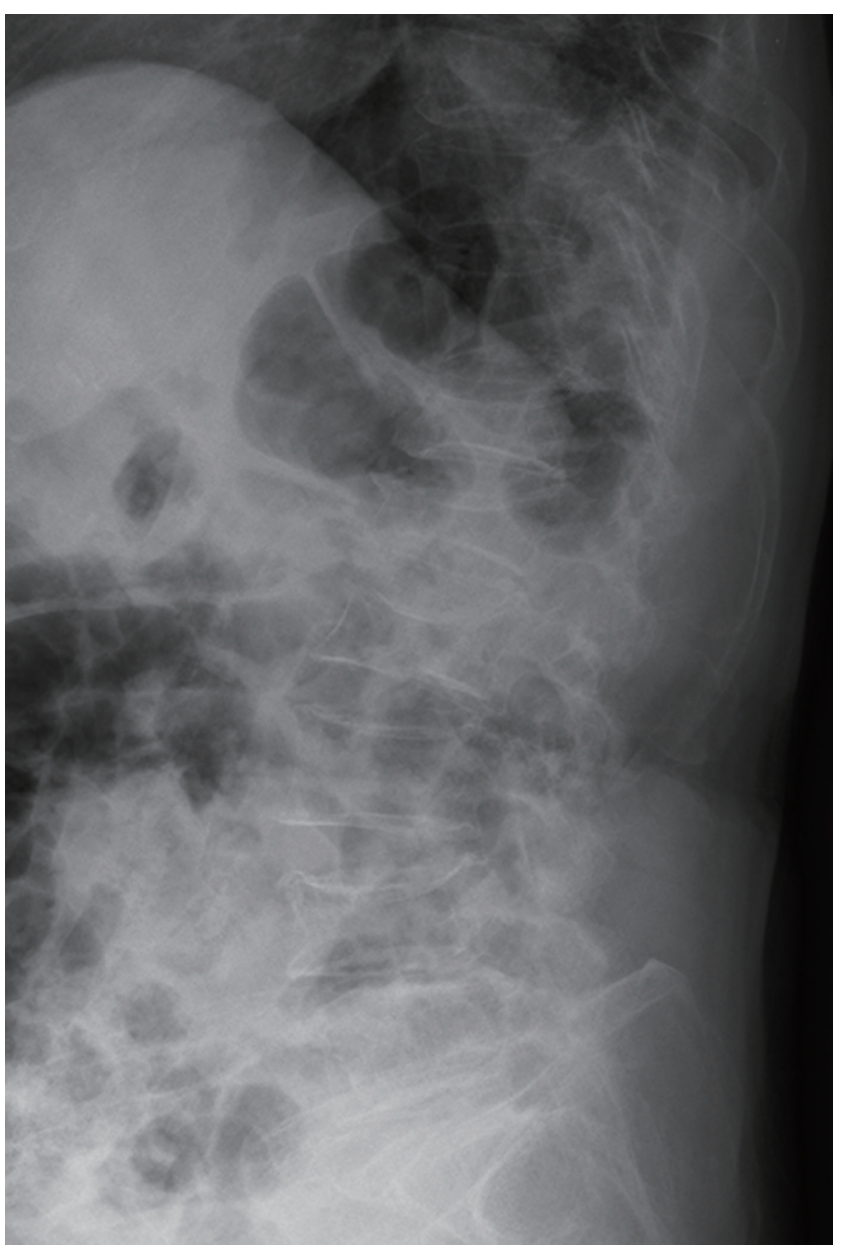

Fig. 2. Simple radiologic finding showed multiple osteoporotic compression fracture in thoracolumbar spine.

later, at the patient's follow-up visit, the VAS score was 5/10 constantly. Eight months later, she felt her back area discomfort again. So we tried Scrambler therapy for four times. After finishing treatment, her pain subsided and the VAS score was 4/10.

\section{DISCUSSION}

The mechanism of scrambler therapy has not been clearly revealed, but relieved acute and chronic pain on the basis of information theory. Scrambler therapy synthesizes 16 different types of artificial nerve action potentials similar to normal nerve impulses, assembles them into sequences, and transmits them to the patient' $s$ nociceptors. And it modulates the pain information through patient-specific cutaneous electro-stimulation [2]. In author's cases, all had been treated with conventional managements for more than several years but still complained of severe pain. For the pur- pose of pain relief, they received Scrambler therapy for 50 minutes daily for 5-10 consecutive days based on some clinical trials [1,2]. All three patients did not complain of any previously experienced severe pain after they received the Scrambler therapy. When the treatment was finished, constant pain was decreased by more than $50 \%$ compared with the previous condition. One of the three patients was given more treatment for a transient aggravation. Since then, all patients have been well managed with oral medications and do not complain of any undesirable side effects. Several studies on the efficacy of Scrambler therapy for cancer and refractory neuropathic pain such as post herpetic neuralgia have been introduced recently and reported good outcomes [1-9]. Scrambler therapy for the treatment of chemotherapy-induced peripheral neuropathy showed 53\% reduction in pain score from baseline, $44 \%$ reduction in tingling, and 37\% reduction in numbness [6]. And Scrambler therapy for cancer associated chronic neuropathic pain provided sustained improvement both acutely and chronically [8]. Marineo et al. [2] described that scrambler therapy relieved chronic neuropathic pain more effectively than guideline-based drug therapy, which $91 \%$ pain reduction compared with a $28 \%$ reduction using medications. Some case reports showed reduction in refractory post herpetic pain [5] and cancer pain [9]. Marineo et al. [2] suggested that this reason may be due to the feeling of new sensation around the dermatome along the lines of nerve transmission, the so called 'pain scramble.' Marineo et al. [2] described that the scrambler therapy provided the following mechanisms. First, Scrambler therapy gives new 'non pain' information that patients report new sensations in the pain area and it is not simple C-fiber electrical stimulation, which would produce pain. Second, scrambler therapy analgesia occurs quickly, suggesting that the receptors are transmitting the 'non pain' information. Third, the sustained pain relief for days or months suggests either resetting of calcium channels or remodulation of the pain system's response.

In conclusion, through this report, the author showed that Scrambler therapy appears to have benefit in the treatment of untreatable pain, who poorly respond on conventional treatments or have a fear of receiving other invasive treatments. The author was able to obtain a satisfactory effect by using scrambler therapy in patients with long-lasting untreatable pain and they did not complain of desirable side effects. However, there is not so many research regarding the influence of Scrambler therapy, so further larger investigation needed including more research with a large number of patients for various types of pain conditions. 


\section{REFERENCES}

1. Marineo G. Untreatable pain resulting from abdominal cancer: new hope from biophysics? JOP 2003;4:1-10.

2. Marineo G, Iorno V, Gandini C, Moschini V, Smith TJ. Scrambler therapy may relieve chronic neuropathic pain more effectively than guidelinebased drug management: results of a pilot, randomized, controlled trial. J Pain Symptom Manage 2012;43:87-95.

3. Sabato AF, Marineo G, Gatti A. Scrambler therapy. Minerva Anestesiol 2005;71:479-82.

4. Smith TJ, Marineo G. Treatment of postherpetic pain with Scrambler therapy, a patient-specific neurocutaneous electrical stimulation device. Am J Hosp Palliat Care 2013 Jul 8 [Epub]. http://dx.doi.org/10.1177/ 1049909113494002.

5. Ko YK, Lee HY, Lee WY. Clinical experiences on the effect of scrambler therapy for patients with postherpetic neuralgia. Korean J Pain 2013;26: 98-101.
6. Pachman DR, Weisbrod BL, Seisler DK, Barton DL, Fee-Schroeder KC, Smith TJ, et al. Pilot evaluation of Scrambler therapy for the treatment of chemotherapy-induced peripheral neuropathy. Support Care Cancer 2015;23:943-51.

7. Pachman DR, Watson JC, Loprinzi CL. Therapeutic strategies for cancer treatment related peripheral neuropathies. Curr Treat Options Oncol 2014;15:567-80.

8. Coyne PJ, Wan W, Dodson P, Swainey C, Smith TJ. A trial of Scrambler therapy in the treatment of cancer pain syndromes and chronic chemotherapy-induced peripheral neuropathy. J Pain Palliat Care Pharmacother 2013;27:359-64.

9. Park HS, Sin WK, Kim HY, Moon JY, Park SY, Kim YC, et al. Scrambler therapy for patients with cancer pain: case series. Korean J Pain 2013;26: 65-71.

10. Starkweather AR, Coyne P, Lyon DE, Elswick RK Jr, An K, Sturgill J. Decreased low back pain intensity and differential gene expression following Calmare $^{\circledR}$ : results from a double-blinded randomized sham-controlled study. Res Nurs Health 2015;38:29-38. 